\title{
Mais do que uma insuficiência de crescimento: relato de caso
}

Sara Magalhães, ${ }^{1}$ Marlene Rodrigues, ${ }^{2}$ Maria João Nabais Sá ${ }^{3}$

\section{RESUMO}

Introdução: A insuficiência de crescimento (IC) constitui um motivo frequente de referenciação a consultas médicas em idade pediátrica. As causas podem ser múltiplas, sendo a sua identificação fundamental para o tratamento e/ou prevenção de complicações secundárias. Apesar da baixa prevalência das doenças genéticas inatas, é importante estar ciente desta possibilidade na investigação etiológica da IC.

Descrição do caso: Apresenta-se o caso de uma criança de dois anos, do género masculino, com antecedentes de restrição do crescimento intrauterino e dificuldades de alimentação desde o período neonatal, observada em consulta de medicina geral e familiar com IC e atraso global do desenvolvimento psicomotor (ADPM).

Adicionalmente, a observação de dismorfias craniofaciais típicas, íris estrelada, voz rouca, laxidez articular e uma personalidade hipersociável e comunicativa, permitiram suspeitar de síndroma de Williams.

Foi solicitada consulta de genética médica, tendo o diagnóstico clínico sido confirmado através da identificação da deleção da região cromossómica 7q11.23, o que permitiu uma vigilância clínica adequada da criança, assim como o aconselhamento genético dos jovens progenitores.

Comentário: Apesar das inúmeras causas de IC, as síndromas de etiologia genética devem ser consideradas na presença de ADPM e/ou anomalias congénitas.

O médico de família, como principal mediador entre o doente e a família, deverá possuir conhecimentos básicos que permitam o reconhecimento de manifestações clínicas sugestivas de doenças genéticas inatas, de modo a efetuar um diagnóstico e/ou uma referenciação precoces dos doentes e seus familiares em risco.

Palavras-chave: Síndroma de Williams; Insuficiência de crescimento, Doenças genéticas inatas.

\section{INTRODUÇÃO}

A insuficiência de crescimento (IC) caracteriza-se pela presença de peso e estatura abaixo do percentil $3\left(\mathrm{P}_{3}\right)$ em, pelo menos, duas avaliações consecutivas ou pelo cruzamento de dois percentis nas curvas de crescimento de referência da população em estudo. ${ }^{1}$ Não existe, no entanto, ainda uma definição consensual e uma das dificuldades da sua abordagem reside na distinção variante do normal versus doença orgânica.

Dependendo da sua intensidade e idade de início, as repercussões no desenvolvimento físico e intelectual da criança podem ser graves, constituindo um motivo

1. Médica Interna de Medicina Geral e Familiar. USF Ponte, ACES Alto Ave - Guimarães

2. Médica Interna de Pediatria. Serviço de Pediatria, Hospital de Braga

3. Médica Interna de Genética Médica. Serviço de Genética Médica, Centro de Genética Médica Dr. Jacinto Magalhães/Centro Hospitalar do Porto frequente de preocupação dos pais e de procura de ajuda médica. De acordo com a literatura, a IC representa 5 a 10\% dos motivos de consulta nos cuidados de saúde primários (CSP) nos EUA. ${ }^{1}$

Relativamente à sua patofisiologia, esta pode dividir-se em causas orgânicas, funcionais ou resultar da interação de ambas (Quadro I). ${ }^{2-4}$ Nos CSP, o inadequado aporte calórico constitui a causa mais prevalente de IC. ${ }^{1}$ Por outro lado, as síndromas de etiologia genética destacam-se de entre as causas orgânicas em idade pré-natal.

A síndroma de Williams constitui uma causa de IC associado a alterações multissistémicas de expressão variável, que incluem uma dismorfia facial típica, cardiopatias, alterações endócrinas, anomalias do tecido conjuntivo, atraso do desenvolvimento psicomotor e características de personalidade, nomeadamente hiperssociabilidade, comunicação e ansiedade extremas. 


\begin{tabular}{|c|c|c|}
\hline Idade & \multicolumn{2}{|l|}{ Diagnóstico } \\
\hline Pré-natal & \multicolumn{2}{|c|}{$\begin{array}{l}\text { Exposição a tóxicos } \\
\text { Infeções pré-natais } \\
\text { Prematuridade } \\
\text { Restrição de crescimento fetal } \\
\text { Síndromas congénitas }\end{array}$} \\
\hline \multirow[t]{2}{*}{ 0-6 Meses } & Causas funcionais & $\begin{array}{l}\text { Alterações psicológicas maternas: depressão/défice de ligação } \\
\text { Aporte inadequado de leite materno; preparação inadequada de leite adaptado } \\
\text { Dificuldades na sucção e deglutição } \\
\text { Más condições socioeconómicas } \\
\text { Recusa alimentar }\end{array}$ \\
\hline & Causas orgânicas & $\begin{array}{l}\text { Acidose tubular renal } \\
\text { Alergia/intolerância às proteínas do leite de vaca } \\
\text { Infeções perinatais } \\
\text { Infeções recorrentes } \\
\text { Doença cardíaca congénita } \\
\text { Doença do refluxo gastroesofágico } \\
\text { Erros inatos no metabolismo } \\
\text { Fibrose quística } \\
\text { Infeção pelo vírus da imunodeficiência humana }\end{array}$ \\
\hline \multirow[t]{2}{*}{ 6-12 Meses } & Causas funcionais & $\begin{array}{l}\text { Alterações psicológicas maternas: ansiedade de separação/individualização } \\
\text { Desconhecimento das necessidades alimentares do lactente } \\
\text { Dificuldades na transição de alimentos sólidos } \\
\text { Más condições sócioeconómicas }\end{array}$ \\
\hline & Causas orgânicas & $\begin{array}{l}\text { Acidose tubular renal } \\
\text { Alergia alimentar } \\
\text { Doença celíaca } \\
\text { Doença do refluxo gastroesofágico } \\
\text { Fibrose quística } \\
\text { Infeção pelo vírus da imunodeficiência humana }\end{array}$ \\
\hline \multirow[t]{2}{*}{$>12$ Meses } & Causas funcionais & $\begin{array}{l}\text { Problemas psicossociais } \\
\text { Dieta inadequada }\end{array}$ \\
\hline & Causas orgânicas & $\begin{array}{l}\text { Alergia alimentar } \\
\text { Doença celíaca } \\
\text { Doenças crónicas } \\
\text { Doença do refluxo gastroesofágico } \\
\text { Fibrose quística } \\
\text { Infeções recorrentes }\end{array}$ \\
\hline
\end{tabular}

Nesta síndroma, fatores pré e pós-natais confluem para a causalidade da IC como a restrição de crescimento intrauterino (RCIU), a dificuldade na sucção, o refluxo gastroesofágico (RGE), a aversão a determinados alimentos e as cólicas (Quadro II). ${ }^{5}$
A síndroma de Williams apresenta uma prevalência de 1/7.500 e resulta de uma microdeleção no braço longo do cromossoma 7 (locus 7q11.23), envolvendo o gene da elastina (ELN) com função estrutural nas fibras elásticas. ${ }^{6}$ Na maioria dos casos é esporádica (pais não afe- 


\section{QUADRO II. Características clínicas da síndroma de Williams ${ }^{5,7}$}

\begin{tabular}{|c|c|c|}
\hline Dismorfia facial & $\begin{array}{l}\text { Face } \\
\text { Olhos } \\
\text { Nariz } \\
\text { Boca e cavidade oral } \\
\text { Pavilhões auriculares }\end{array}$ & $\begin{array}{l}\text { Fronte alta, estreitamento bitemporal, hipoplasia da região malar, mandíbula } \\
\text { pequena, face longa na idade adulta } \\
\text { Estrabismo, íris estrelada, proeminência do tecido periorbitário, epicanto } \\
\text { Ponte nasal achatada, nariz curto com narinas antevertidas } \\
\text { Filtro longo, boca grande, com lábios espessos, dentes pequenos e espaçados, } \\
\text { má-oclusão } \\
\text { Lóbulos grandes com pavilhões auriculares proeminentes }\end{array}$ \\
\hline Crescimento & \multicolumn{2}{|c|}{$\begin{array}{l}\text { Nascimento pós-termo (idade gestacional }>41 \text { semanas) } \\
\text { Insuficiência de crescimento (peso e estatura }<\text { P5) } \\
\text { Refluxo gastroesofágico ou vómitos } \\
\text { Irritabilidade por cólicas }>4 \mathrm{M} \\
\text { Obstipação crónica }\end{array}$} \\
\hline $\begin{array}{l}\text { Comportamento e } \\
\text { desenvolvimento }\end{array}$ & \multicolumn{2}{|c|}{$\begin{array}{l}\text { Personalidade hiperafável } \\
\text { Ansiedade/défice de atenção } \\
\text { Hipersensibilidade aos sons/hipoacusia } \\
\text { Défices construtivos visuoespaciais } \\
\text { Atraso na aquisição da fala e posterior discurso abundante } \\
\text { Atraso de desenvolvimento psicomotor }\end{array}$} \\
\hline Cardíacos & \multicolumn{2}{|c|}{$\begin{array}{l}\text { Estenose supravalvular aórtica } \\
\text { Estenose pulmonar periférica } \\
\text { Hipertensão } \\
\text { Sopros cardíacos } \\
\text { Outras malformações congénitas }\end{array}$} \\
\hline Tecido conjuntivo & \multicolumn{2}{|c|}{$\begin{array}{l}\text { Voz rouca } \\
\text { Ombros descaídos } \\
\text { Laxidez articular } \\
\text { Pele laxa e macia } \\
\text { Hérnia inguinal } \\
\text { Diverticulose intestinal e vesical } \\
\text { Prolapso retal }\end{array}$} \\
\hline Endócrinos & \multicolumn{2}{|l|}{$\begin{array}{l}\text { Hipercalcemia } \\
\text { Hipercalciúria } \\
\text { Hipotiroidismo }\end{array}$} \\
\hline
\end{tabular}

tados). Quando herdada, apresenta transmissão autossómica dominante, com risco de recorrência de $50 \%$ na descendência dos indivíduos afetados. ${ }^{6}$

Os autores descrevem a investigação diagnóstica de uma criança com síndroma de Williams, centrando-se no doente e na família. Este artigo pretende sensibilizar para a necessidade de o médico de família possuir conhecimentos básicos sobre síndromas de etiologia genética associadas a IC, destacando a importância da abordagem multidisciplinar para uma precoce e adequada orientação clínica do doente e dos seus familiares, o que permite prevenir investigação e ansiedade desnecessárias no doente e na família.

\section{DESCRIÇÃO DO CASO}

Rapaz de dois anos de idade, de raça caucasiana pertencente a uma família reconstruída da classe média, na fase III do ciclo de vida de Duvall e fruto de uma re- 
lação não consanguínea. Primeiro filho de uma mulher de 31 anos, ex-fumadora de 5,25 UMA (Unidades MaçoAno), com antecedentes pessoais de asma moderadamente controlada e com irmã com trissomia 21. O pai, homem de 38 anos sem antecedentes pessoais ou familiares relevantes, tinha um filho saudável de nove anos, de uma relação prévia.

Gravidez desejada e planeada, de baixo risco, vigiada simultaneamente pelo médico de família (MF) e obstetra privado por opção da grávida e, desde a fase pré-concecional, não apresentando intercorrências até às 33 semanas de idade gestacional, momento em que foi detetada restrição de crescimento intrauterino (RCIU) simétrica. Foi efetuado ecocardiograma fetal, onde se verificou "discreta hiperrefringência da válvula tricúspide, sem alteração do fluxo ou funcionamento cardíaco". Foram excluídas outras malformações, assim como positividade para serologias TORCH.

Nascimento às 38 semanas de gestação e seis dias por parto distócico por trabalho de parto estacionário, com Apgar 9/10. Antropometricamente apresentava peso de $2,105 \mathrm{~kg}\left(<\mathrm{P}_{3}\right)$, comprimento de $43,7 \mathrm{~cm}\left(<\mathrm{P}_{3}\right)$ e perímetro cefálico de $31 \mathrm{~cm}\left(<\mathrm{P}_{3}\right)$. Não se verificaram intercorrências no período neonatal, sendo o rastreio de doenças metabólicas negativo. Efetuou aleitamento materno exclusivo desde o período neonatal e até aos 5,5 meses, com destaque de uma anorexia que se mantinha desde o nascimento. Fez suplementação com vitamina $\mathrm{D}$ durante o primeiro ano de vida com cumprimento do Programa Nacional de Vacinação e imunização extra-plano para Pneumococo.

Após o nascimento e no contexto da investigação de RCIU, em consulta de neonatologia, foi efetuada ecografia transfontanelar que se revelou normal. Ainda neste contexto, em consulta de cardiologia pediátrica, no primeiro mês de vida, realizou ecocardiograma onde se verificou a presença de estenose fisiológica da artéria pulmonar com gradiente de $12 \mathrm{mmHg}$, considerada normal para a idade. A ecografia reno-vesical detetou ligeira pielectasia bilateral, motivando vigilância em consulta de nefrologia pediátrica. A repetição de ecografia reno-vesical aos nove meses, sem anomalias estruturais, permitiu concluir tratar-se de uma pielectasia transitória mas que, por intercorrência de pielonefrite nesta idade, motivou manutenção em consulta de nefrologia pediátrica até aos 17 meses.
Manteve paralelamente, e desde o nascimento, consulta com o seu MF cumprindo a periodicidade estabelecida no Plano Nacional de Saúde Infantil e Juvenil. Aos seis meses em consulta com o MF foi detetada criptorquidia unilateral esquerda com exclusão de outras anomalias testiculares por ecografia.

Iniciou diversificação alimentar aos 5,5 meses sem registo de alergias ou intolerâncias alimentares. Mantinha, contudo, uma anorexia que a família veio progressivamente a interpretar como "característica de uma criança difícil” (sic). A mãe descrevia que os momentos de alimentação se revelaram desde o nascimento momentos de grande sofrimento para todos os elementos da família: "a criança passava horas ao peito sem sucção e com recusa inicial na pega" (sic). A manutenção da situação gerou nesta, progressivamente, um sentimento de culpa, considerando-se com limitadas competências para o aleitamento materno, assim como com leite "de baixo conteúdo nutricional" (sic). Apesar da desmistificação destes factos pelos profissionais de saúde, a mãe compensava a sua inquietação com o prolongamento dos tempos de alimentação e encurtamento do seu intervalo. A avó materna, também prestadora de cuidados, descrevia que a criança "nunca chorava com fome e, quando se forçava a alimentação, esta recusava e demorava longos períodos para comer pequenas quantidades" (sic). Por volta dos 12 meses iniciou um quadro de obstipação que era controlado com medidas dietéticas.

Adicionalmente, na sequência de episódios de broncospasmo recorrente com início aos 6,5 meses de vida foi referenciado para consulta de imunoalergologia, onde foi observado aos 13 meses. Foram excluídas alergias respiratórias e alimentares, fibrose quística, doença celíaca, assim como doença parasitária intestinal. A totalidade da investigação revelou-se normal, à exceção da presença de um hipotiroidismo subclínico. Aos 22 meses, em consulta de pediatria/gastroenterologia-nutrição, foi também pedido cariótipo e estudo metabólico extenso, que se revelaram normais. Durante este período, o crescimento estaturo-ponderal manteve-se inferior ao $\mathrm{P}_{3}$ mas paralelamente a esta curva de crescimento, tendo sido interpretado como normal num contexto de RCIU.

No que refere ao desenvolvimento psicomotor, adquiriu controlo cervical entre os dois-três meses, apresentou sorriso social aos três meses, sentou com apoio 


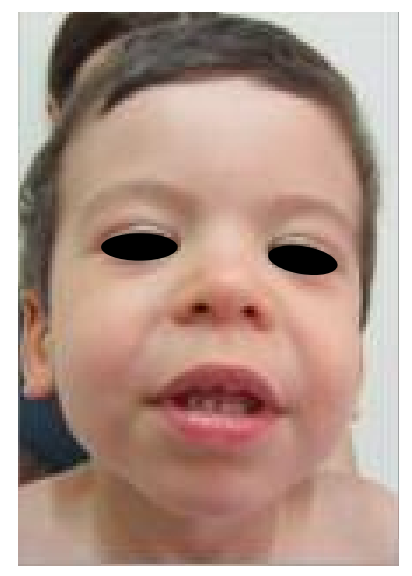

Figura 1. Fotografia da criança, observada aos dois anos, mostrando características craniofaciais típicas de síndroma de Williams, nomeadamente estreitamento bitemporal, abundância de tecido periorbitário, epicanto bilateral, bochechas proeminentes, nariz curto, com ponta bolbosa e narinas antevertidas, filtro longo, boca larga com lábios grossos, pavilhões auriculares com lobos proeminentes.

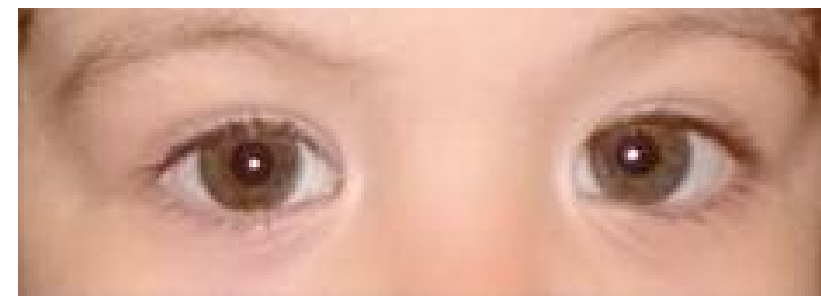

Figura 2. Íris estrelada presente na criança descrita com síndroma de Williams.

entre os seis-sete meses e sem apoio aos oito meses e meio, efetuou pinça aos nove meses, palrou aos 13 meses e iniciou marcha sem apoio aos 19 meses.

Por razões de conveniência geográfica mudou de centro de saúde, tendo sido observado pela primeira vez pelo novo MF de família aos dois anos e três meses. Ao exame físico apresentava antropometria no $\mathrm{P}_{3}$, dismorfia facial e não similar a nenhum dos progenitores (estreitamento bitemporal, abundância de tecido periorbitário, epicanto bilateral, bochechas proeminentes, nariz curto, com ponta bolbosa e narinas antevertidas, filtro longo, boca larga com lábios grossos, pavilhões auriculares com lobos proeminentes - Figura 1), íris estrelada (Figura 2), voz rouca, laxidez articular e uma personalidade anormalmente sociável e comunicativa para a idade e para um primeiro contacto. Alertado pela preocupação mantida dos pais relativamente à IC e no contexto da sua experiência prévia em genética médica, referenciou para esta especialidade com suspeita de síndroma de Williams.

Foi observado aos dois anos e quatro meses, tendo-se confirmado o diagnóstico clínico através do exame citogenético de FISH (Fluorescent in situ hibridization), que identificou a deleção da região cromossómica 7q11.23. Foi ainda excluída a mesma alteração genética nos progenitores, concluindo tratar-se de uma situação de novo, com risco de recorrência $<1 \%$ em novo filho do casal de progenitores (isto é, baixo, embora ligeiramente superior ao da população geral). Foi elaborada estratégia de follow-up com envio de relatório para o MF e para as especialidades de seguimento: pediatria do desenvolvimento (para vigilância do atraso de desenvolvimento psicomotor [ADPM]), endocrinologia pediátrica (por hipotiroidismo subclínico), ORL (para rastreio de hipoacusia), oftalmologia (para rastreio de hiperopia), ortopedia (para rastreio de alterações esqueléticas), cirurgia pediátrica (para vigilância de criptorquidia unilateral esquerda), cardiologia pediátrica (para reavaliação cardíaca) e a gastrenterologia pediátrica (por obstipação). Manteve ainda seguimento em consulta de psicologia no Centro de Genética Médica (para avaliação do ADPM). A criança encontra-se atualmente em vigilância pelas especialidades, não se tendo verificado o aparecimento de outras malformações. No que se refere ao desenvolvimento estaturo-ponderal apresenta-se no $P_{50}$ de peso e estatura, nas curvas de crescimento adaptadas a síndroma de Williams.

Enquadrando-se a IC no contexto desta síndroma com implicações no normal crescimento da criança, foi decidido iniciar suplementação com hormona tiroideia (levotiroxina $0,25 \mathrm{ug} / \mathrm{dia}$ ) e monitorização anual da sua função. No que se refere ao desenvolvimento psicomotor foi iniciada terapia da fala, terapia ocupacional, musicoterapia, natação e apoio educacional com ativação das equipas locais de intervenção. Assim, aos três anos e onze meses eram já evidentes melhorias em todas as áreas do desenvolvimento avaliadas pela escala de Desenvolvimento Mental de Griffiths, nomeadamente ao nível da linguagem expressiva e compreensiva, assim como da autonomia/sociabilidade. No que se refere à avaliação familiar, após exclusão do caráter hereditário da condição, foi realizado aconselhamento genético com tranquilização dos progenitores, no sentido de ausência de risco elevado de repetição da patologia numa segunda gestação do casal.

\section{COMENTÁRIO}

No caso descrito, a presença de IC associada a um conjunto de características clínicas permitiu que o MF 
levantasse a hipótese diagnóstica de síndroma de Williams. Esta síndroma genética, com manifestações multissistémicas e comuns a outras entidades nosológicas, nomeadamente RCIU, IC e ADPM, diferencia-se pela sua associação a uma dismorfia craniofacial típica. ${ }^{6}$

Apesar de a IC ser motivo frequente de avaliação em idade pediátrica e apresentar na maioria dos casos um curso benigno, ${ }^{3}$ quando em associação com outras anomalias congénitas e ADPM deve fazer suspeitar de uma causa genética. $\mathrm{O}$ atraso na obtenção do diagnóstico constituiu uma fonte geradora de ansiedade na família com sofrimento associado a processos que se pretendiam vinculativos, como a amamentação. Adicionalmente, o atraso no diagnóstico, em especial no contexto de uma doença genética, pode condicionar graves repercussões no que concerne ao prognóstico do doente, assim como na prevenção do risco de recorrência familiar.

O MF, beneficiando de um contacto privilegiado com os diferentes membros da família e do seu acompanhamento longitudinal, deverá estar sensibilizado para a identificação das características dismórficas que se distinguem de variantes do normal ou de traços familiares. ${ }^{6}$ Neste caso particular, o MF, com experiência prévia na área da genética médica, desempenhou um papel vital no reconhecimento dessa dismorfia e na sua integração com as restantes anomalias que não pareciam enquadrar-se num caso isolado de IC.

O diagnóstico de síndroma de Williams durante uma fase precoce da infância mostrou enormes benefícios não só para a criança como para a família: evicção de investigação excedentária e muitas vezes invasiva; início mais precoce do tratamento do hipotiroidismo com benefícios quer a nível estatuto-ponderal quer cognitivo, este último potenciado pela intervenção precoce; monitorização adequada das complicações da doença; assim como disponibilização de aconselhamento genético aos progenitores que viam, até ao momento, o receio de uma nova gravidez pelo risco de recorrência da doença. Do mesmo modo, a avaliação das repercussões na funcionalidade da família não deve ser esquecida, devendo ser fomentadas atitudes preventivas nas consultas, no sentido de lidar com a ansiedade, dúvidas e frustrações familiares. Perante a ausência de fenótipo típico e da deleção causadora de síndroma de Williams nos pais foi possível estimar um risco baixo de recorrência desta patologia numa futura gravidez do casal, permitindo reduzir a sua ansiedade face ao planeamento familiar. No entanto, dado não se puder excluir a existência de rearranjos cromossómicos equilibrados nos pais, foi recomendada uma consulta pré-concecional em genética médica com possibilidade de diagnóstico pré-natal numa futura gravidez. Por outro lado, a possibilidade de fertilidade em doentes com síndroma de Williams fez antecipar a necessidade de aconselhamento genético ao doente, numa fase em que este planeie ter filhos, pelo risco de recorrência de $50 \%$ na sua descendência. ${ }^{6}$

Salienta-se ainda que, apesar do cariótipo se revelar uma ferramenta importante na investigação inicial de crianças com IC ou atraso desenvolvimento psicomotor, em muitos casos não é suficiente para a obtenção do diagnóstico, tal como se verificou no caso descrito. ${ }^{5}$ Assim, a abordagem médica multidisciplinar ao nível do diagnóstico ou seguimento constitui a melhor solução para o utente e família. Numa estrutura de prestação de cuidados em pirâmide, os efeitos serão tanto mais potenciados quanto maiores forem os conhecimentos e meios da base da pirâmide, na qual se encontram os cuidados de saúde primários.

Assim, do presente caso concluiu-se que o MF deve ter presente a possibilidade de uma doença genética aquando na presença de IC de início pré-natal e em associação com outras anomalias congénitas e ADPM. Perante um elevado grau de suspeição de patologia genética deverá referenciar atempadamente e estabelecer a articulação necessária entre as especialidades, tendo presente que as patologias genéticas envolvem geralmente necessidades multidisciplinares.

\section{REFERÊNCIAS BIBLIOGRÁFICAS}

1. Cole SZ, Lanham JS. Failure to thrive: an update. Am Fam Physician. 2011;83(7):829-34.

2. Emond A, Drewett R, Blair P, Emmett P. Postnatal factors associated with failure to thrive in term infants in the Avon Longitudinal Study of Parents and Children. Arch Dis Child. 2007;92(2):115-9.

3. Olsen EM, Skovgaard AM, Weile B, Petersen J, Jørgensen T. Risk factors for weight faltering in infancy according to age at onset. Paediatr Perinat Epidemiol. 2010;24(4):370-82.

4. Kirkland RT, Motil KJ, Duryea TK. Failure to thrive (undernutrition) in children younger than two years: etiology and evaluation [Internet]. UpToDate; 2015 [updated 2016 Dec]. Available from: http://www.uptodate.com/contents/failure-to-thrive-undernutrition-in-children-younger-than-two-years-etiology-and-evaluation 
5. Preus M. The Williams syndrome: objective definition and diagnosis. Clin Genet. 1984;25(5):422-8.

6. Morris CA. Williams syndrome. In: Pagon RA, Adam MP, Ardinger HH, Wallace SE, Amemiya A, Bean L], et al, editors. GeneReviews ${ }^{\circledR}[$ Internet]. Seattle,WA: University of Washington; 1993-2016 [updated 2013 Jun 13].Available from: http://www.ncbi.nlm.nih.gov/books/NBK1249/.

7. Committee on Genetics. American Academy of Pediatrics: health care supervision for children with Williams syndrome. Pediatrics. 2001;107(5):1192-204.

\section{CONFLITOS DE INTERESSE}

As autoras referem não ter quaisquer conflitos de interesse.

\section{ENDEREÇO PARA CORRESPONDÊNCIA}

Sara Magalhães

E-mail: saraimaga@gmail.com

Recebido em 20-03-2016

Aceite para publicação em 25-11-2016

\section{ABSTRACT}

\section{MORE THAN FAILURE TO THRIVE: A CASE REPORT}

Introduction: Failure to thrive is a common reason for referral of children for pediatric consultation. There are many causes and the correct diagnosis is essential for the treatment and prevention of secondary complications. Although genetic diseases as a cause of failure to thrive are rare, awareness of this possibility is necessary.

Case report: A 2-year-old boy, with history of intrauterine growth retardation and feeding difficulties from the neonatal period, was seen in a general practice consultation due to failure to thrive and global psychomotor developmental delay.

In addition to these medical problems, the child had craniofacial dysmorphism, a stellate iris pattern, a hoarse voice, lax joints, and an overfriendly and talkative personality. The diagnosis of Williams syndrome was proposed.

The patient was referred to a medical genetics consultation and the diagnosis was confirmed by demonstrating a chromosomal microdeletion at 7q11.23. The child was referred for appropriate follow-up of the child and his young parents received genetic counselling.

Comment: The presence of psychomotor developmental delay and multiple physical anomalies should raise the possibility of a genetic syndrome as a cause of failure to thrive.

Family physicians should have basic knowledge of genetic disorders to allow the recognition of clinical signs suggestive of inborn genetic diseases in order to enable early diagnosis and referral of the patient and the family for medical genetic consultation.

Keywords: Williams syndrome; Failure to thrive; Inborn genetic diseases. 